\title{
Stimulation of Synaptic Vesicle Exocytosis by the Mental Disease Gene DISC1 is Mediated by N-Type Voltage-Gated Calcium Channels
}

\section{OPEN ACCESS}

Edited by:

Stéphane Martin, IPMC CNRS UMR7275 - University of Nice Sophia antipolis, France

Reviewed by: Timothy A. Ryan, Weill Medical College of Cornell University, USA

Yann Humeau,

Centre National de la Recherche Scientifique (CNRS), France

${ }^{*}$ Correspondence: Marc Fivaz

marc.fivaz@duke-nus.edu.sg

tThese authors have contributed equally to this work

Received: 01 February 2016 Accepted: 31 May 2016 Published: 14 June 2016

Citation: Tang W, Thevathasan JV, Lin Q, Lim KB, Kuroda K, Kaibuchi K Bilger $M$, Soong TW and Fivaz $M$ (2016) Stimulation of Synaptic Vesicle Exocytosis by the Mental Disease Gene DISC 1 is Mediated by N-Type Voltage-Gated Calcium Channels. Front. Synaptic Neurosci. 8:15. doi: 10.3389/fnsyn.2016.00015

\begin{abstract}
Willcyn Tang ${ }^{1 \dagger}$, Jervis Vermal Thevathasan ${ }^{1 \dagger}$, Qingshu Lin ${ }^{2}$, Kim Buay Lim ${ }^{1}$, Keisuke Kuroda ${ }^{3}$, Kozo Kaibuchi ${ }^{3}$, Marcel Bilger ${ }^{4}$, Tuck Wah Soong ${ }^{2}$ and Marc Fivaz ${ }^{1,2 *}$

${ }^{1}$ DUKE-NUS Medical School, Program in Neuroscience and Behavioral Disorders, Singapore, Singapore, ${ }^{2}$ Department of Physiology, Yong Loo Lin School of Medicine, National University of Singapore, Singapore, Singapore, ${ }^{3}$ Department of Cell Pharmacology, Nagoya University Graduate School of Medicine, Nagoya, Japan , ${ }^{4}$ DUKE-NUS Medical School, Program in Health Services and Systems Research, Singapore, Singapore
\end{abstract}

Lesions and mutations of the DISC1 (Disrupted-in-schizophrenia-1) gene have been linked to major depression, schizophrenia, bipolar disorder and autism, but the influence of DISC1 on synaptic transmission remains poorly understood. Using two independent genetic approaches-RNAi and a DISC1 KO mouse-we examined the impact of DISC1 on the synaptic vesicle (SV) cycle by population imaging of the synaptic tracer $\mathrm{VGpH}$ in hippocampal neurons. DISC1 loss-offunction resulted in a marked decrease in SV exocytic rates during neuronal stimulation and was associated with reduced $\mathrm{Ca}^{2+}$ transients at nerve terminals. Impaired SV release was efficiently rescued by elevation of extracellular $\mathrm{Ca}^{2+}$, hinting at a link between DISC1 and voltage-gated $\mathrm{Ca}^{2+}$ channels. Accordingly, blockade of N-type Cav2.2 channels mimics and occludes the effect of DISC1 inactivation on SV exocytosis, and overexpression of DISC1 in a heterologous system increases Cav2.2 currents. Collectively, these results show that DISC1-dependent enhancement of SV exocytosis is mediated by Cav2.2 and point to aberrant glutamate release as a probable endophenotype of major psychiatric disorders.

Keywords: DISC1, hippocampus, glutamate, psychiatric disorders, schizophrenia, neurotransmitter, synaptic vesicle release

\section{INTRODUCTION}

Genome-wide association studies and exome sequencing efforts have led to the identification of hundreds of variants associated with psychiatric disorders (International Schizophrenia et al., 2009; Moskvina et al., 2009; Glessner et al., 2010; Fromer et al., 2014; Schizophrenia Working Group of the Psychiatric Genomics, 2014), confirming the complex polygenic nature of these diseases. These genomic data also revealed a significant overlap in genes or gene networks associated with distinct mental illnesses (Cross-Disorder Group of the Psychiatric Genomics et al., 2013), suggesting a common genetic and perhaps circuitry basis for major psychiatric disorders. However, the synaptic and circuitry defects underlying these disorders remain poorly defined, hindering the development of therapeutic solutions. 
DISC1 is the prototypical example of a gene associated with several major psychiatric disorders. It was discovered in a Scottish family at the site of a balanced chromosomal translocation that strongly segregates with major depression, schizophrenia and bipolar disorder (St Clair et al., 1990; Millar et al., 2000, 2001). The high penetrance $(\sim 70 \%)$ of this translocation for mental illness supports a causal link between this genetic lesion and major psychiatric conditions (Chubb et al., 2008). DISC1 variants (haplotypes, single nucleotide polymorphisms and copy number variations) have since been independently associated with depression, schizophrenia, bipolar disorders and autism spectrum disorders (Ekelund et al., 2001, 2004; Sachs et al., 2005; Kilpinen et al., 2008). Thus, DISC1 is a major susceptibility factor for mental illness and a relevant genetic entry point to identify core endophenotypes implicated in neuropsychiatric disorders.

The translocation breakpoint in this Scottish family is located in the C-terminal portion of DISC1 and results in overall reduced expression of the full-length transcript and protein (Millar et al., 2005), suggesting that haploinsufficiency is the main mechanism by which this chromosomal alteration confers risk to disease. Alternatively, it has been proposed that a C-terminal truncated form of DISC1 is expressed from the translocated allele and may be pathogenic (Hikida et al., 2007; Pletnikov et al., 2008), although expression of the truncated DISC1 protein in translocation carriers remains to be demonstrated. Consistent with a disease mechanism based on DISC1 loss-of-function, DISC1 expression is also attenuated in human induced pluripotent stem (iPS) cells derived from members of a family with a DISC1 frame-shift mutation that co-segregates with major psychiatric disorders (Wen et al., 2014).

The identification of a large DISC1 interactome consisting of proteins belonging to different ontologic families suggests broad functions of DISC1 in nerve cells. Accordingly, DISC1 has been implicated in multiple aspects of neuronal and brain development, including neurogenesis (Clapcote et al., 2007; Shen et al., 2008; Mao et al., 2009; Singh et al., 2010; Lee et al., 2011), neuronal migration (Kamiya et al., 2005; Duan et al., 2007; Kubo et al., 2010; Steinecke et al., 2012) and maturation (Duan et al., 2007; Shinoda et al., 2007; Niwa et al., 2010). Even though DISC1 interacts with several signaling proteins known to regulate synaptic functions, relatively little is known about functions of DISC1 at the synapse. In particular, the impact of DISC1 on neurotransmitter release remains largely unexplored, despite the fact that aberrant dopamine and glutamate neurotransmission is a probable cause of schizophrenia and other mood disorders (Howes et al., 2015).

Given the preferential expression of DISC1 in the hippocampus and the involvement of this brain structure in cognition and psychiatric disorders (Chubb et al., 2008), we set out to determine the impact of DISC1 on synaptic vesicle (SV) cycling in hippocampal neurons. We used two independent genetic strategies to alter DISC1 expression-RNAi and a DISC1 KO mouse-and imaged SV cycling and $\mathrm{Ca}^{2+}$ dynamics in large synapse populations. We show that DISC1 elevates synaptic $\mathrm{Ca}^{2+}$ signals and boosts $\mathrm{SV}$ exocytosis at glutamatergic terminals. Our results further indicate that $\mathrm{N}$-type voltage-gated $\mathrm{Ca}^{2+}$ channels (VGCCs) mediate the stimulatory effect of DISC1 on SV release. These findings identify a central role of DISC1 in neurotransmitter release and provide new insights on the biological basis of synaptic dysfunction in major psychiatric disorders.

\section{MATERIALS AND METHODS}

\section{DNA, shRNA Constructs, Lentiviruses and Antibodies}

The pCAGGs vGlut1-pHluorin (vGpH) and pCAGGs Synaptophysin-GCaMP3 (SyGC3) constructs were gifts from R. Edwards (UCSF; Voglmaier et al., 2006) and S. Voglmaier (UCSF; Li et al., 2011). The pFUGW (Addgene \#14883) shRNAexpressing lentiviral vector was modified to express mCherry (pFUmChW). The shRNA targeting sequences are as follows: (1) Scramble 5'GGAGCAGACGCTGAATTAC3' (Kamiya et al., 2005); (2) DISC1-E 5'GGCTACATGAGAAGCACAG3' (exon 2; Duan et al., 2007); and (3) DISC1-A 5'GGAAGG GCTAGAGATGTTC3' (exon 9) designed with Block-it shRNA from Invitrogen. pFUGW scramble shRNA was a gift from A. Sawa (Johns Hopkins). The DISC1 shRNAs constructs were cloned by introducing double-stranded DNA oligos into lentiviral vector pll3.7 (Addgene \#11795) using the HpaI and XhoI sites. DNA fragments containing the U6 promoter and shRNAs were then PCR amplified and cloned into pFUmChW using the PacI site. The human DISC1 gene $\mathrm{L}$ variant (NCBI Refseq NM018662.2) was PCR amplified from pCMV6-XL5 DISC1-tGFP (Origene) and cloned into pIRES2-DsRed-Express (Clontech) using NheI and SmaI sites. All constructs were sequenced before use. Lentiviral particles expressing pFUmChW shRNAs were prepared as described in Tiscornia et al. (2006). The DNA constructs used for wholecell patch clamp recording are as follows: Cav2.1 (generated in T. W. Soong's lab), Cav2.2 (Addgene \#26568), GFP- $\beta_{2 a}$ and $\alpha_{2} \delta_{1}$ (kindly provided by T. Snutch, UBC). The rabbit polyclonal Abs against Cav2.1 (\#ACC-001) and Cav2.2 (\#ACC002) were from Alomone Labs. The polyclonal $\mathrm{Ab}$ against the C-terminus of mouse DISC1 was previously described (Kuroda et al., 2011). The polyclonal Ab against human DISC1 (ab59017) and monoclonal $\mathrm{Ab}$ against bassoon were from Abcam (ab82958).

\section{Mouse Lines, Primary Neuron Cultures and Transfection Protocols}

DISC1 $(\Delta 2-3)$ mice (C57BL/6JJmsSlc) have been described before Kuroda et al. (2011). Heterozygous DISC1 $1^{w t / \Delta 2-3}$ mice were crossed to each other to obtain $D I S C 1^{\mathrm{wt} / \mathrm{wt}}$ and $D I S C 1^{\Delta 2-3 / \Delta 2-3}$ lines from which hippocampal neurons were prepared from E17/E18 embryos, according to published procedures using papain (Worthington) digestion (Huettner and Baughman, 1986). Primary rat hippocampal neurons were prepared from E18 embryos according to Kaech and 
Banker (2006) and as previously described in Garcia-Alvarez et al. (2015). For live-cell imaging and immunocytochemistry experiments, neurons were grown on poly-L-Lysine coated glass coverslips, on top of a glial feeder according to the Banker Protocol (Kaech and Banker, 2006). For biochemical analysis, cells were seeded on poly-L-Lysine coated 6-well culture dishes. Unless otherwise stated, neurons were cultured for 14-16 days. The vGpH and SyGC3 constructs were electroporated in freshly-dissociated neurons using the Nucleofector kit (Amaxa Biosystems, Lonza). Lentiviral particles expressing shRNAs were added on DIV2, at a MOI (multiplicity of infection) of 1-3. All animal procedures were approved by the SingHealth Institutional Animal Care and Use Committee (IACUC) of Singapore.

\section{Live-Cell Confocal Imaging and Immunofluorescence}

Time-lapse confocal imaging was performed on an inverted Eclipse TE2000-E microscope (Nikon, USA), mounted with a spinning-disk confocal scan head (CSU-10; Yokogawa, Japan), and equipped with a temperature controlled $\left(36.5^{\circ} \mathrm{C}\right)$ stage and an autofocusing system (PFS; Nikon). Images were acquired with an Orca-Flash 4.0 CCD camera (Hamamatsu Photonics, Japan) controlled by MetaMorph 7.8.6 (Molecular Devices, CA, USA) at $0.5 \mathrm{~Hz}$ or $20 \mathrm{~Hz}$. Samples were imaged using a $60 \times$ (NA 1.4) objective in Tyrode's buffer (150 mM NaCl, $2.5 \mathrm{mM} \mathrm{KCl,} 1 \mathrm{mM} \mathrm{CaCl}, 1 \mathrm{mM} \mathrm{MgCl}_{2}$, $6 \mathrm{mM}$ glucose, $25 \mathrm{mM}$ HEPES, $\mathrm{pH}$ 7.4) supplemented with $25 \mu \mathrm{M}$ 6-cyano-7-nitroquinoxaline-2,3-dione/CNQX (Tocris Bioscience) and $50 \mu \mathrm{M}$ D,L-2-amino-5-phosphonovaleric acid/AP5 (Tocris Bioscience). Coverslips were mounted in an RC-21BRFS chamber (Warner Instruments, USA) equipped with platinum wire electrodes. Field stimulation was induced by a square pulse stimulator (Grass Technologies, USA) and monitored by an oscilloscope (TDS210, Tektronix, USA). Trains of action potentials (APs) were generated by applying $20 \mathrm{~V}$ pulses (1 ms duration) at 10 or $20 \mathrm{~Hz}$. Our typical stimulation paradigm for $\mathrm{vGpH}$ measurements involved two consecutive trains of 300 APs at $10 \mathrm{~Hz}$, separated by $\sim 5 \mathrm{~min}$ to allow synapses to recover. $\mathrm{vGpH}$ responses between the first and second stimulation were highly reproducible (data not shown). For measuring SV exocytic rates, the second stimulation was preceded (30 s earlier) by the addition of Bafilomycin A1 (Baf; AG scientific, USA) $0.5 \mu \mathrm{M}$. To normalize for total expression of $\mathrm{vGpH}$ in each individual bouton, $50 \mathrm{mM} \mathrm{NH}{ }_{4} \mathrm{Cl}$ was added at the end of the time series. For SyGC3 measurements, $\mathrm{Ca}^{2+}$ signals were normalized by adding $10 \mu \mathrm{M}$ or $50 \mu \mathrm{M}$ (for Figure 4G) ionomycin (Sigma-Aldrich) at the end of the stimulation protocol. $\omega$-agatoxin TK125 nM (Tocris Bioscience) and $\omega$-conotoxin GVIA $125 \mathrm{nM}$ (Alomone Labs) were used for experiment involving inhibition of $\mathrm{P} / \mathrm{Q}$-type $\mathrm{Ca}^{2+}$ channels and $\mathrm{N}$-type $\mathrm{Ca}^{2+}$ channels activity, respectively. For immunofluorescence studies, neurons grown on glass coverslips were fixed in $4 \%$ paraformaldehyde with $4 \%$ sucrose in PBS and permeabilized with $100 \mathrm{ng} / \mathrm{ml}$ Digitonin
(Sigma-Aldrich). Cells were then incubated with 5\% goat serum to block non-specific binding sites and stained with the primary and Alexa Fluor-conjugated secondary antibodies (Life Technologies). Coverslips were then mounted on glass slides and imaged with an inverted laser scanning confocal microscope (LSM710, Zeiss) with a Plan-Apochromat $63 \times(\mathrm{NA}=1.40)$ objective.

\section{Image Analysis}

For automated analysis of $\mathrm{vGpH}$ responses, we wrote a Matlab script that segments responsive boutons based on the difference between peak $\mathrm{vGpH}$ intensity during the first stimulation and baseline intensity prior to stimulation $\left(\Delta F=F_{\text {peak }}-F_{\text {baseline }}\right)$. An intensity threshold for $\Delta F$ was selected to resolve individual boutons and exclude those with $\Delta F$ below $5 \%$. The same threshold was used for all conditions in one independent experiment (i.e., one neuron preparation with control vs. DISC1-depleted conditions). This threshold value was minimally adjusted (less than 10\% change) across all independent experiments described in this article. Binarized boutons were then slightly dilated (Figure 1B) to ensure that $\mathrm{vGpH}$ fluorescence was captured in its totality even after minor lateral movement or change in shape. Time series with minor $x-y$ drifts (originating from the stage) were re-aligned using a script previously described (Thevathasan et al., 2013). Segmented boutons were then size gated, with gating parameters kept constant across all experiments. $\mathrm{vGpH}$ fluorescence intensity was then extracted in each segmented bouton across the time series and divided by the signal after $\mathrm{NH}_{4} \mathrm{Cl}$ to normalize for $\mathrm{vGpH}$ expression (Figure 1C). This segmentation strategy ensures that the same boutons are analyzed during the two consecutive AP trains. $\mathrm{vGpH}$ traces with significant baseline drifts between the first and second stimulation or after Baf application were excluded. A similar segmentation approach was used to analyze SyGC3 $\mathrm{Ca}^{2+}$ signals. SV exocytic rates were obtained by measuring the slope of the $\mathrm{vGpH}$ rise during the second stimulation (in the presence of Baf). The first six time points (during stimulation) were used for linear regression analysis. To measure endocytic rates, the $\mathrm{vGpH}$ trace during the first stimulation was subtracted from that obtained during the second stimulation. The resulting trace is a measure of endocytosis (Figure 1D). Endocytic rates were measured by linear fitting of six time points chosen after stimulation onset when endocytosis kicks in. Presynaptic localization, abundance and density of Cav2.1 or Cav2.2 were analyzed with a modified version of a Matlab script described previously (Poon et al., 2014). All Matlab scripts are available upon request.

\section{Immunoblotting}

Cultured primary neurons or HEK293T cells were washed with ice-cold PBS and lysed with RIPA buffer (10 mM Tris$\mathrm{HCl} \mathrm{pH}=7.2,150 \mathrm{mM} \mathrm{NaCl}, 1 \%$ TritonX-100, 0.1\% SDS, $5 \mathrm{mM}$ EDTA, $0.25 \%$ Na-deoxycholate) supplemented with Complete protease inhibitor and PhosphoStop phosphatase inhibitor (Roche). For analysis of hippocampal tissue, the 


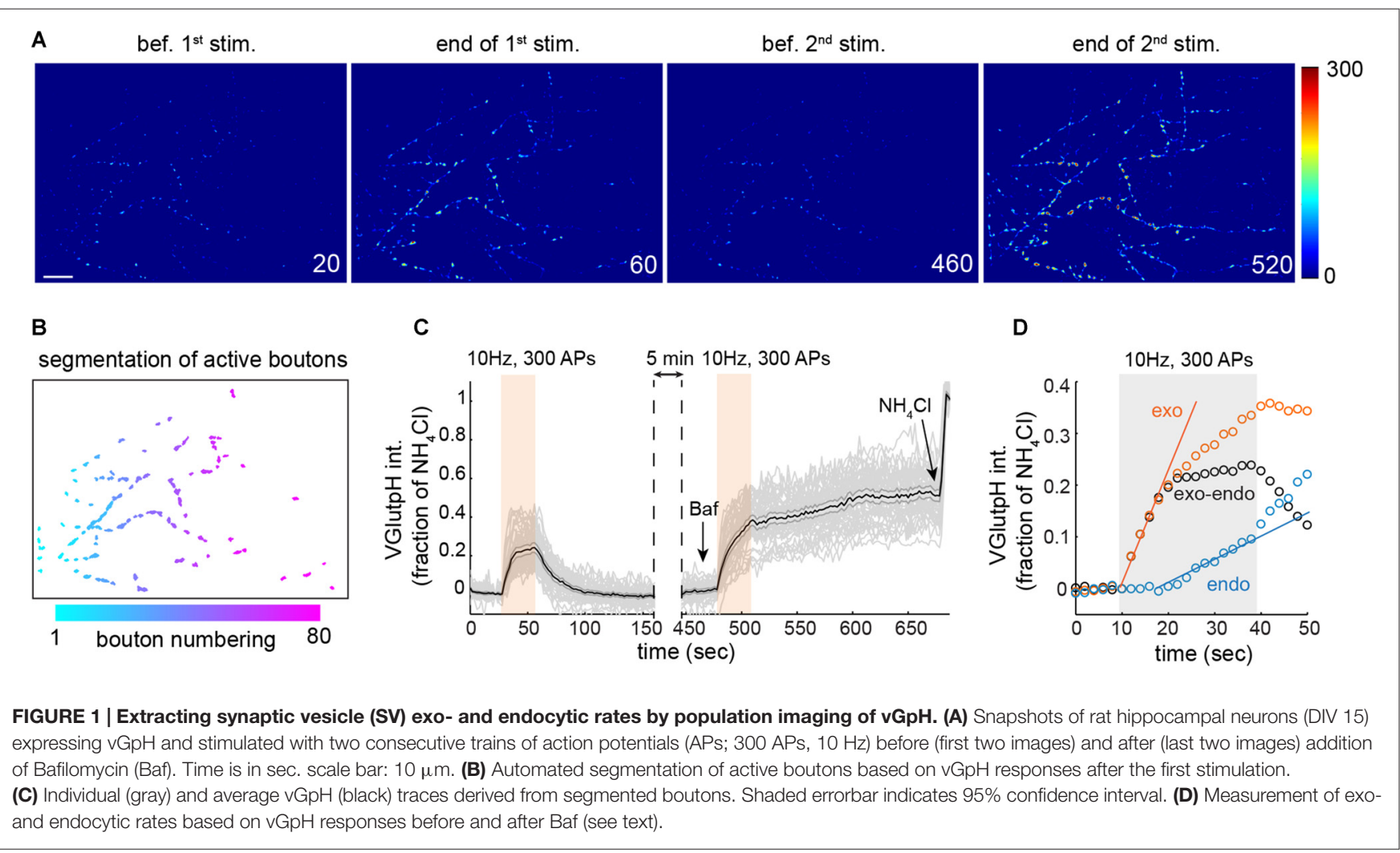

hippocampi from P10 mice were harvested and homogenized in RIPA buffer using a Dounce tissue homogenizer. Lysates were cleared by centrifugation and boiled in Laemmli sample buffer. Equal amount of total proteins were loaded. Samples were then analyzed by SDS-PAGE, transferred onto nitrocellulose membranes, probed with appropriate primary and HRP-labeled secondary antibodies and revealed by enhanced chemiluminescence.

\section{Whole-Cell Patch Clamp Recordings of Cav2.1 and Cav2.2 Currents}

Cav2.1 or Cav2.2, the auxiliary subunits (GFP- $\beta_{2 a}$ and $\alpha_{2} \delta_{1}$ ) and DISC1 were transiently transfected in HEK 293 cells using the calcium phosphate method (Huang et al., 2012). Whole-cell patch-clamp recordings were performed within 36-72 $\mathrm{h}$ after transfection. The external solution contained (in mM) 10 HEPES, 140 TEA- $\mathrm{MeSO}_{3}$ and $5 \mathrm{BaCl}_{2}$ (pH 7.4, 300-310 mOsm). The glass pipette solution was backfilled with pipette solution (in $\mathrm{mM}) 10$ HEPES, $5 \mathrm{CsCl}, 138 \mathrm{Cs}-\mathrm{MeSO}_{3}, 0.5 \mathrm{EGTA}, 1 \mathrm{MgCl}_{2}, 2$ $\mathrm{mg} / \mathrm{ml} \mathrm{MgATP}$ ( $\mathrm{pH} 7.3,290-300$ mOsm). HEK 293 cells were held at $-90 \mathrm{mV}$ using the Axopatch $700 \mathrm{~B}$ amplifier (Axon Instruments). The series resistance for all recordings was less than $5 \mathrm{M} \Omega ; 70-80 \%$ compensation on serial resistance and cell membrane capacitance were applied. A $\mathrm{P} / 4$ protocol was used to subtract leakage current. All recordings were obtained with an Axon Digidata 1440A data acquisition system, sampled at $5-50 \mathrm{kHz}$ and low pass-filtered at $1 \mathrm{kHz}$ or $6 \mathrm{kHz}$. The $\mathrm{I}-\mathrm{V}$ curves were obtained from $10 \mathrm{mV}$ voltage-steps ranging from -50 to $40 \mathrm{mV}$ and fitted with a modified Boltzmann equation:

$$
I=G_{\mathrm{max}}\left(E_{\mathrm{rev}}-V\right) /\left(1+e^{\frac{V_{1 / 2 \mathrm{act}}-V}{k_{\mathrm{act}}}}\right)
$$

where, $I=$ current density (in $\mathrm{pA} / \mathrm{pF}$ ), $G_{\max }=$ maximum conductance (in $\mathrm{nS} / \mathrm{pF}$ ), $E_{\mathrm{rev}}=$ reversal potential, $V=$ measured potential, $V_{1 / 2 \text { act }}=$ midpoint voltage for current activation, and $k_{\text {act }}=$ the slope factor.

We used a tail protocol to measure current density; cells were depolarized using $10 \mathrm{mV}$ voltage steps, from -60 to $60 \mathrm{mV}$. Following depolarization, tail currents were evoked with a $10 \mathrm{~ms}$ pulse at $-50 \mathrm{mV}$. The data were fitted with single Boltzmann equation:

$$
I=I_{\min }+\left(I_{\max }-I_{\min }\right) /\left(1+e^{\frac{V_{1 / 2 \text { inact }}-V}{k_{\text {inact }}}}\right)
$$

where, $I_{\max }$ and $I_{\min }=$ maximal and minimal current respectively, $V_{1 / 2 \text { inact }}=$ the half-maximal voltage for current inactivation, $k_{\text {inact }}=$ slope of inactivation curve.

\section{Statistics}

The experimental design of this study implies that data collected at individual synaptic terminals are clustered according to neuron preparations and imaged fields. Galbraith et al. (2010) demonstrated that such clustering can adversely affect statistical inference when not accounted for. In order to 
probe for clustering effects (i.e., intra-field correlations), we tested whether bouton responses significantly vary from field to field. For this, we conducted Wald tests of the null hypothesis of no difference in mean outcome across fields within each condition, which revealed ( $p$-value $<0.0001)$ strong intra-field correlation. To account for these correlations, we performed our statistical analysis in the framework of linear mixed models (Laird and Ware, 1982) with normallydistributed random field effects and preparation fixed effects. In experiments involving one genetically-modified condition and one control group, we tested the null hypothesis of no difference between the mean outcome of the groups via 2 -sample $t$-tests. In experiments involving two geneticallymodified conditions and one control group, we tested the null hypothesis of no difference between the mean outcome of each group and the control group jointly using Wald tests. All tests were performed at the 5\% level of statistical significance and carried out using the statistical software Stata version 13.2. Because our statistical approach (linear mixed model) is not a standard practice when analyzing synaptic properties, we compared the p-values obtained with our method with those measured by the more common field averaging approach, where the information of a field is collapsed to a single independent observation by taking the mean of bouton responses. Both methods are valid and gave comparable results (Supplementary Table T1), although the field averaging approach is less statistically efficient as it diminishes the information that can be obtained from the data by reducing individual measurements in a field to one observation (Galbraith et al., 2010).

\section{RESULTS}

\section{DISC1 Loss-of-Function Slows Down SV Cycling}

We opted for an imaging approach based on the synaptic tracer $\mathrm{vGpH}$ to explore the impact of DISC1 on the SV cycle. $\mathrm{vGpH}$ consists of the $\mathrm{pH}$-sensitive GFP variant pHluorin (Miesenbock et al., 1998) fused to the SV-resident glutamate transporter vGlut1. pHluorin, which faces the acidic lumen of SVs, undergoes a $\sim 20$-fold increase in fluorescence intensity when exposed to the neutral $\mathrm{pH}$ of the extracellular milieu after membrane fusion. (Sankaranarayanan et al., 2000). Following glutamate discharge and $\mathrm{vGpH}$ re-uptake, SVs are rapidly re-acidified and $\mathrm{vGpH}$ fluorescence is quenched. This property has made $\mathrm{vGpH}$ a valuable tool to monitor both exo- and endocytosis of SVs at single synapses.

Due to the inherent variability in the properties of individual synaptic boutons (Ariel et al., 2013) we measured SV cycling in large synapse populations. For this, we developed an image analysis algorithm that identifies all responding boutons in a given field (Figures 1A,B), and imaged 16-18 fields from at six independent rat hippocampal neuron cultures, yielding close to a thousand synaptic boutons for each condition. We employed this algorithm to monitor SV cycling in response to a stimulation paradigm that consists of two consecutive trains of APs (Fernandez-Alfonso and Ryan, 2004; Burrone et al., 2006). The amplitude of the first $\mathrm{vGpH}$ transient is governed by the relative rates of SV exo- and endocytosis during stimulation (Figure 1C). To separate the contributions of exo- and endocytosis, we blocked SV re-acidification with the vacuolar $\mathrm{H}^{+}$ATPase inhibitor Bafilomycin (Baf) during the second stimulation, which allows selective measurement of SV exocytosis (Figure 1C). To account for cell-to-cell variation in $\mathrm{vGpH}$ expression, we normalized each trace based on the $\mathrm{vGpH}$ signal measured after $\mathrm{NH}_{4} \mathrm{Cl}$ addition (Figure 1C). The endocytic component of SV cycling was then computed by subtracting the first $\mathrm{vGpH}$ transient from the $\mathrm{vGpH}$ response after Baf treatment (Figure 1D). Synaptic traces with baseline drifts between the first and second stimulation or after Baf addition were excluded from the analysis (see "Materials and Methods" Section). Exocytosis largely dominates at the onset of stimulation, while endocytosis kicks in towards the end of the AP train. Exo- and endocytic rates were finally obtained by linear fitting of the exo- and endo traces (Figure 1D). Exocytic rates are about three times higher than endocytic rates under this stimulation protocol. To evaluate the variability of bouton responses across fields and neuron preparations, we compiled exocytic rates from six independent experiments (Supplementary Figure S1). Statistical analysis of these responses revealed substantial field to field variation (see "Materials and Methods" Section), implying that the information collected from each bouton does not constitute an independent measurement. To compare synaptic responses in different fields and conditions we used a statistical approach that accounts for correlations within fields and variations in neuron preparations (see "Materials and Methods" Section and Supplementary Table T1).

We then silenced the DISC1 gene using a published shRNA sequence (shRNA-E), targeting exon 2 (Duan et al., 2007) and a new shRNA sequence (shRNA-A) targeting exon 9. These two shRNAs target regions of the DISC1 gene that are fully conserved in rodents and are thus expected to silence rat and mouse DISC1 with the same efficiency. To probe the effect of these shRNAs on DISC1 protein levels, we used an antibody raised against the C-terminus of mouse DISC1, which has previously been validated in a DISC1 KO mouse (Kuroda et al., 2011). In our hands, this antibody poorly reacts with rat DISC1 (not shown). In mouse hippocampal neurons, however, it detects one major protein at $\sim 100 \mathrm{kDa}$ corresponding to the predicted full length DISC1 (Kuroda et al., 2011) and which is substantially reduced in both shRNA-E and -A transduced cells (Figure 2A).

Next, we probed the impact of these shRNAs on the SV cycle in rat hippocampal neurons. We initially chose to work with rat, rather than mouse neurons, because we can harvest substantially more cells from a rat embryonic litter. vGpH signals in shRNA-E and -A expressing neurons display a slower rise and lower amplitude during the first and second stimulation, relative to control cells expressing a scramble shRNA (Figure 2B). Analysis of exocytosis shows 


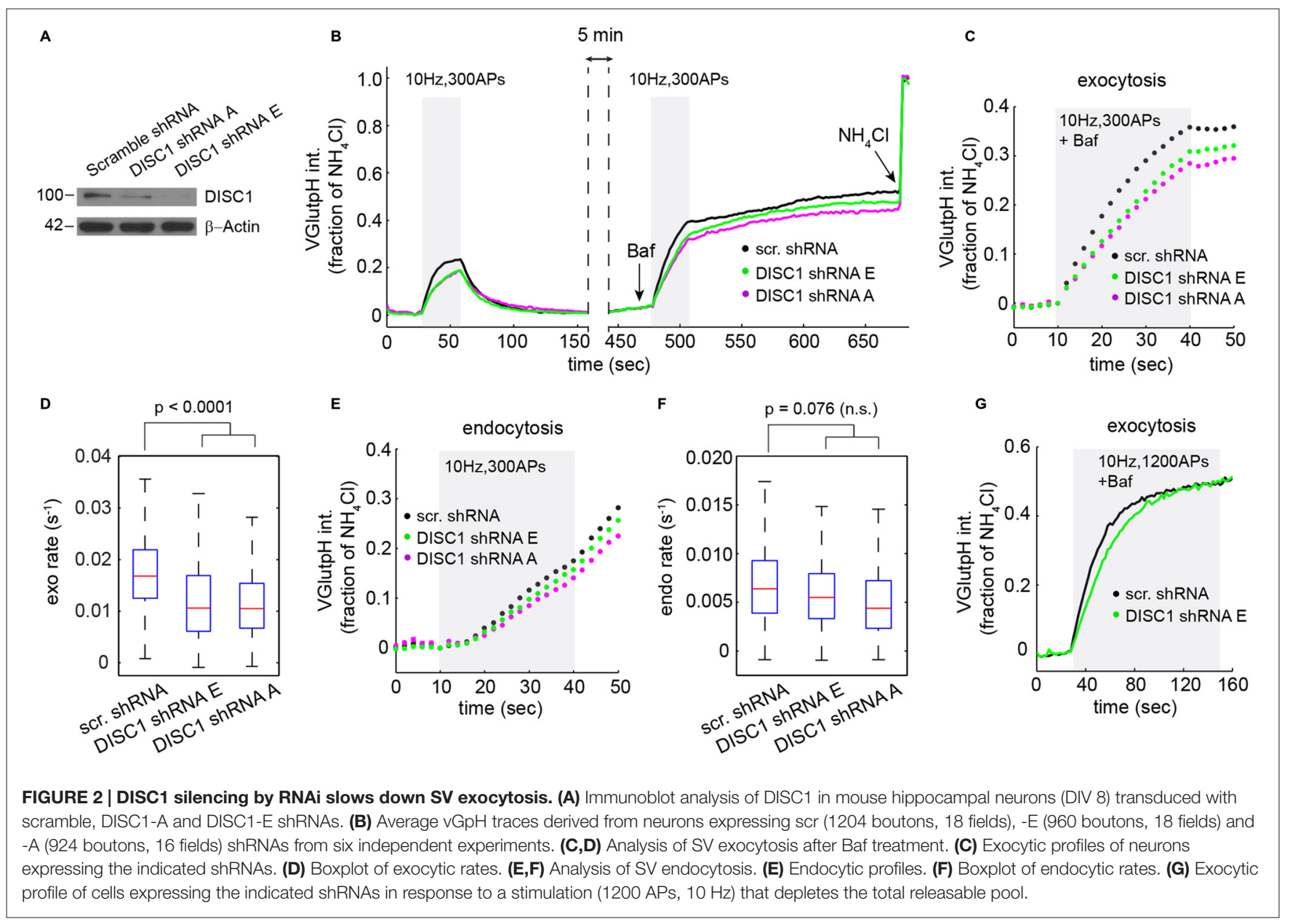

substantial synapse-to-synapse variation in all conditions, but reveals a marked decrease in exocytic rates (Figures 2C,D) and amplitudes (Figure 2C) in both shRNA-E and -A expressing neurons. DISC1 silencing also resulted in slightly slower SV endocytosis, although this effect did not reach statistical significance (Figures 2E,F).

Reduced amplitude of the exocytic response prompted us to test whether DISC1 also regulates the size of the total releasable pool. For this, we applied a stimulus strong enough $(1200 \mathrm{APs}$ at $10 \mathrm{~Hz}$ ) to maximally deplete releasable SVs from presynaptic terminals (Ariel and Ryan, 2010). Under this stimulation regime, $\mathrm{vGpH}$ responses reached the same plateau in DISC1-silenced and control neurons, albeit with different kinetics, arguing against an effect on the total releasable pool but confirming the stimulatory function of DISC1 in SV exocytosis (Figure 2G).

To rule out the possibility of RNAi off-target effects-shRNA$\mathrm{E}$ has recently been suggested to inhibit neuron migration in the developing cortex independently of DISC1 (Tsuboi et al., 2015)-we examined SV cycling in a DISC1 KO mouse that lacks exons 2 and 3 of the DISC1 gene (Kuroda et al., 2011). Homozygous $D I S C 1^{\Delta 2-3 / \Delta 2-3}$ mice show no detectable levels of the major isoform of DISC1 (Figure 3A). Hippocampal neurons derived from $D I S C 1^{\Delta 2-3 / \Delta 2-3}$ mice display SV cycling defects that are remarkably similar to these observed with RNAi. The rates and amplitudes of exocytic responses were substantially reduced relative to $\mathrm{wt}$ cells (Figures 3B-D), while endocytic rates were marginally, but not significantly diminished (Figures 3E,F). Together, these results show that both genetic ablation and RNAi knockdown of DISC1 selectively disrupts SV exocytosis at glutamatergic synapses with no detectable impact on the total releasable pool.

\section{DISC1 Stimulates $\mathrm{Ca}^{2+}$ Influx and Regulates Cav2.2-Dependent SV Release}

Because SV exocytosis is initiated by $\mathrm{Ca}^{2+}$ influx through VGCCs, we measured evoked $\mathrm{Ca}^{2+}$ transients at presynaptic terminals using the SV-targeted $\mathrm{Ca}^{2+}$ sensor SyGC3 (Li et al., 2011). $\mathrm{Ca}^{2+}$ signals evoked by 300 APs $(10 \mathrm{~Hz})$ or $200 \mathrm{APs}(20 \mathrm{~Hz})$ show a rapid rise and partial decay during the stimulus (Figures $\mathbf{4 A}, \mathbf{B}, \mathbf{D}, \mathbf{E}$ ), as observed by others ( $\mathrm{Li}$ et al., 2011). These $\mathrm{Ca}^{2+}$ transients were significantly reduced in shRNA-E and -A expressing neurons, under the same stimulation conditions used for $\mathrm{vGpH}$ measurements (Figures $\mathbf{4 A}, \mathbf{C}$ ), or in response to a higher frequency stimulus (Figure 4B). Similarly, 


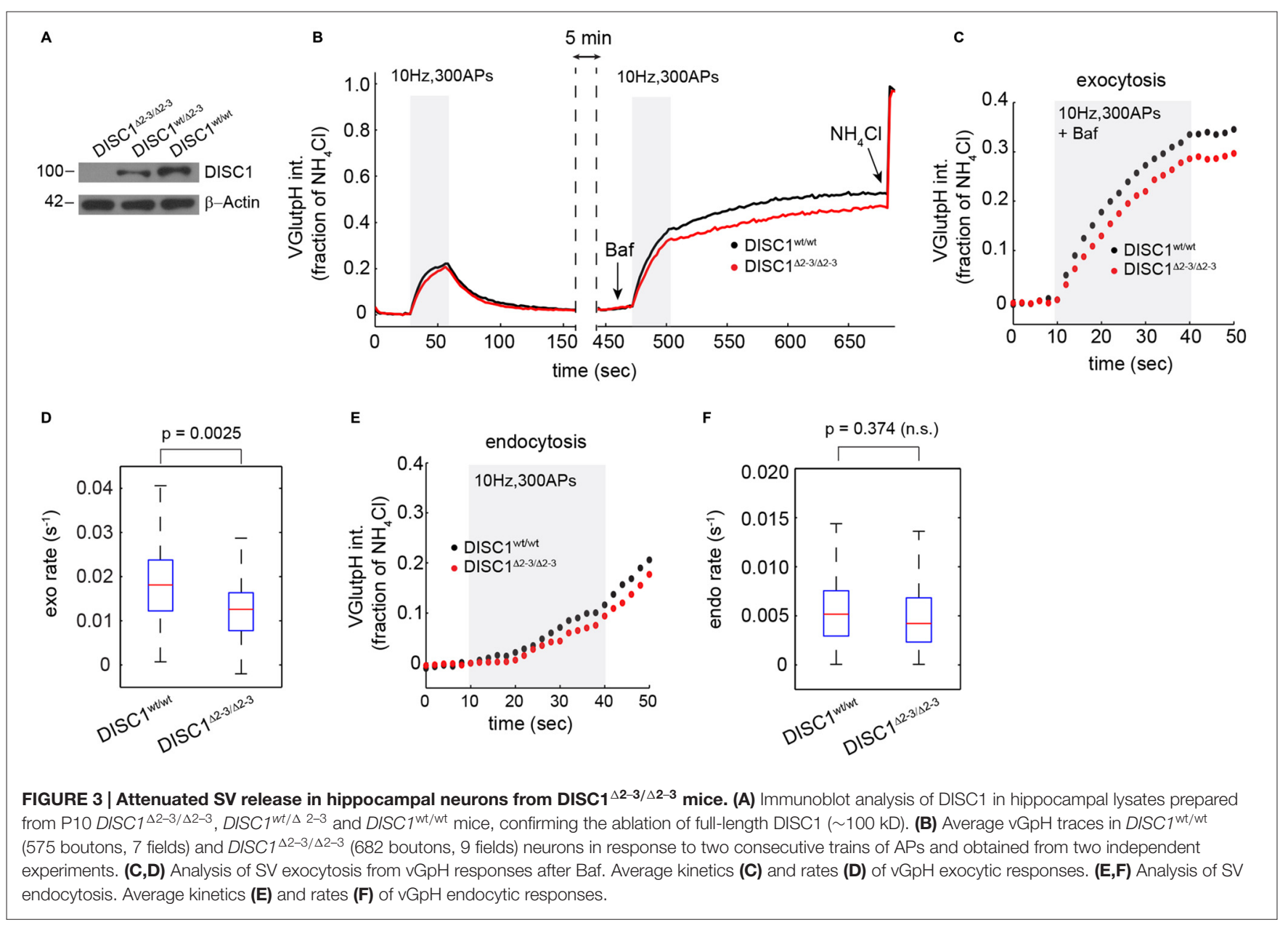

DISC1 $1^{\Delta 2-3 / \Delta 2-3}$ neurons show lowered $\mathrm{Ca}^{2+}$ transients compared to wt cells (Figures 4D-F). We were concerned that, under such prolonged stimulations, $\mathrm{Ca}^{2+}$ concentration in terminals might reach levels that partially saturate SyGC3. $\mathrm{Ca}^{2+}$ signals were therefore also examined in response to shorter stimulations (20 APs, $20 \mathrm{~Hz})$ under conditions well below SyGC3 saturation (Tian et al., 2009; Akerboom et al., 2012). A clear decrease in the amplitude of $\mathrm{Ca}^{2+}$ transients was also observed in DISC1-silenced neurons under this stimulation regime (Figure 4G). Together, these $\mathrm{Ca}^{2+}$ imaging data hint at a $\mathrm{Ca}^{2+}$ influx defect in DISC1inactivated cells, although abnormal clearance of $\mathrm{Ca}^{2+}$ from the presynaptic terminal could also be involved (see "Discussion" Section).

To determine whether reduced $\mathrm{Ca}^{2+}$ influx is the primary cause of SV cycling defects, we attempted to rescue SV exocytosis in DISC1-silenced neurons by elevating extracellular $\mathrm{Ca}^{2+}$ concentration. Shifting extracellular $\mathrm{Ca}^{2+}$ concentration from 2 to $4 \mathrm{mM}$ increases AP-induced $\mathrm{Ca}^{2+}$ entry (Ariel and Ryan, 2010) and restored the $\mathrm{vGpH}$ response (Figure $4 \mathbf{H}$ ), in line with a role of DISC1 in facilitating $\mathrm{Ca}^{2+}$ influx.

At central synapses, the P/Q-type (Cav2.1) and N-type (Cav2.2) $\mathrm{Ca}^{2+}$ channels are the main sources of $\mathrm{Ca}^{2+}$ for initiation of SV exocytosis (Catterall et al., 2013). We used channel-specific toxins to determine the contribution of each subtype to SV release in hippocampal neurons. $\omega$ Conotoxin GVIA (an N-type blocker) reduced SV exocytic rates by $73 \pm 5 \%$ (Figures $\mathbf{5 A}, \mathbf{B}$ ), while $\omega$-Agatoxin TK (a P/Q-type blocker) resulted in a $42 \pm 3 \%$ decrease in SV exocytic rates (Figures 5C,D), in a good agreement with a recent study (Ariel et al., 2013). Note that in these experiments, exocytic rates were approximated by measuring the initial slope of the $\mathrm{vGpH}$ response in the absence of Baf (see Figure 1D). Importantly, blockade of Cav2.2 almost completely occluded the effect of DISC1 silencing on SV exocytosis (Figures 5A,B,E). Blockade of Cav2.1, on the other hand, slightly increased the inhibitory impact of DISC1 knockdown on SV release rates (Figures 5C-E). We conclude from these results that DISC1 selectively stimulates Cav2.2-dependent SV release at hippocampal synapses.

We then asked whether DISC1 regulates Cav2.2 activity by controlling its presynaptic localization and/or abundance. Co-localization studies of endogenous Cav2.2 with the active zone marker bassoon revealed extensive presence of Cav2.2 in synaptic boutons, both in control and DISC1-silenced neurons (Supplementary Figures S2A-C). We found no significant difference between these two groups in the fraction of boutons 

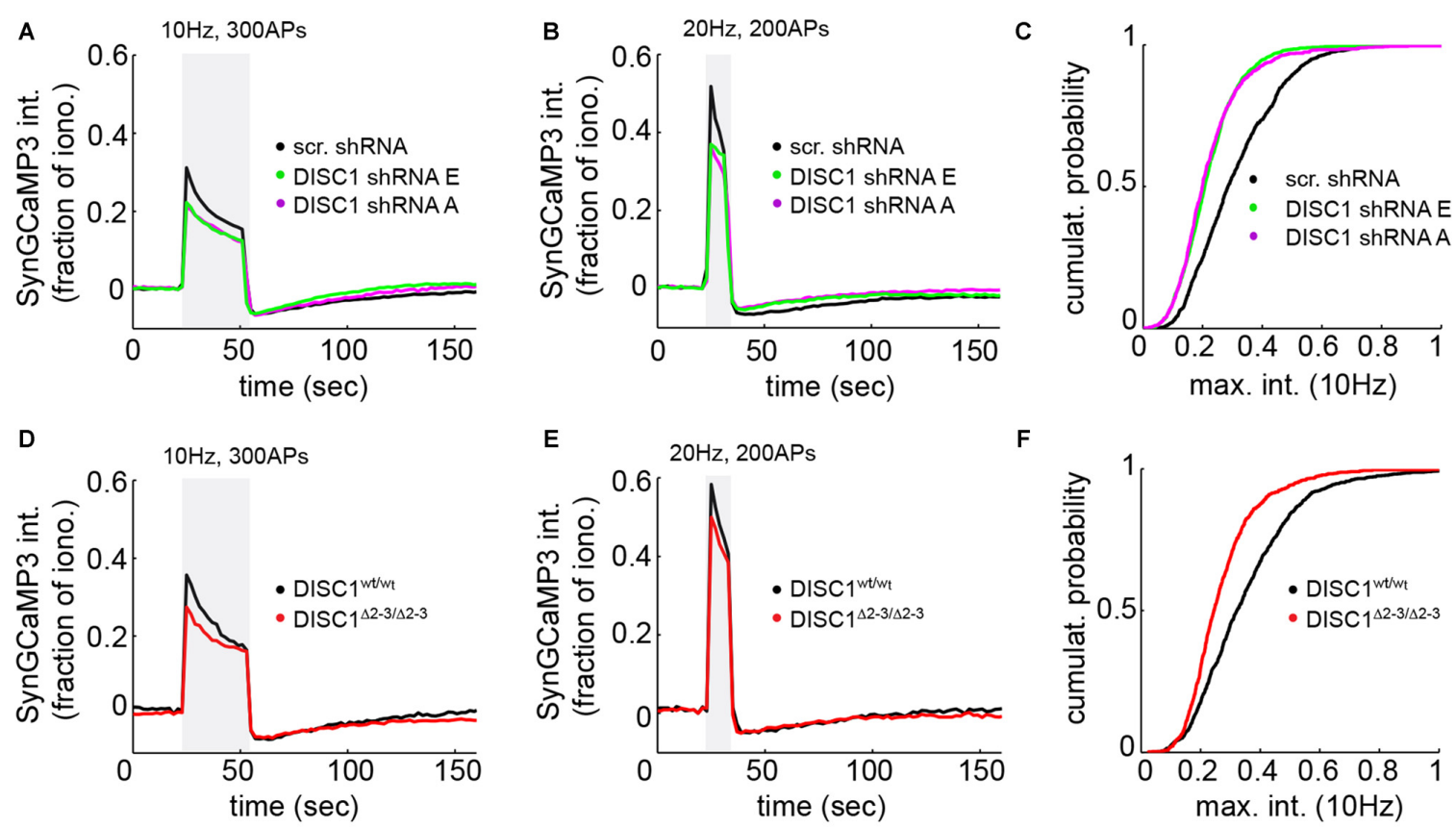

G

H

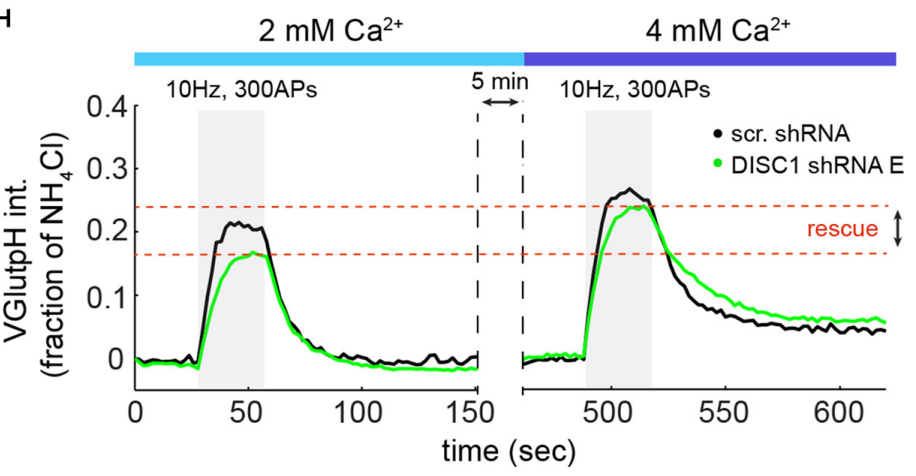

FIGURE 4 | DISC1 loss-of-function reduces evoked $\mathrm{Ca}^{2+}$ transients at nerve terminals. SyGC3 imaging in rat hippocampal neurons (DIV14-16) in response two different trains of APs. (A) Average $\mathrm{Ca}^{2+}$ transients in neurons expressing scr (1052 boutons, 15 fields, 5 experiments (exps)), DISC1-E (1836 boutons, 9 fields, 3 exps) and -A (754 boutons, 11 fields, 3 exps) shRNAs, in response to 300 APs, $10 \mathrm{~Hz}$. The DISC1-E and DISC-A groups are significantly different than the scr group $(p=0.0057)$. (B) Average $\mathrm{Ca}^{2+}$ transients in neurons expressing scr ( 861 boutons, 9 fields, 5 exps), DISC1-E (2118 boutons, 7 fields, 3 exps) and -A (1549 boutons, 9 fields, 3 exps) shRNAs, in response to 200 APs, $20 \mathrm{~Hz}$. (C) Cumulative probability of SyGC3 peak intensity from individual boutons corresponding to (A). (D) Average $\mathrm{Ca}^{2+}$ transients in DISC1 wt/wt (1090 boutons, 11 fields, 3 exps) and DISC1 ${ }^{\Delta 2-3 / \Delta 2-3}$ (727 boutons, 9 fields, 3 exps) neurons, in response to 300 APs, $10 \mathrm{~Hz}$. The DISC1 wt/wt and DISC1 ${ }^{\Delta 2-3 / \Delta 2-3}$ groups are statistically different $\left(p=0.0182\right.$ ). (E) Average $\mathrm{Ca}^{2+}$ transients in DISC1 wt/wt (1365 boutons, 9 fields, 3 exps) and DISC1 $1^{\Delta 2-3 / \Delta 2-3}$ (1233 boutons, 8 fields, 3 exps) neurons, in response to $200 \mathrm{APs}, 20 \mathrm{~Hz}$. (F) Cumulative probability of SyGC3 peak intensity from individual boutons corresponding to (D). (G) Average $\mathrm{Ca}^{2+}$ transients in neurons expressing scr (290 boutons, 6 fields, 2 exps), and DISC1-E (390 boutons, 6 fields, 2 exps) shRNAs, in response to $20 \mathrm{APs}, 20 \mathrm{~Hz}$. The scr and DISC1-E groups are statistically different $(p=0.0036)$. (H) Average vGpH traces in scr (180 boutons, 5 fields, 2 exps) and DISC1-E (488 boutons, 6 fields, 2 exps) shRNA-expressing neurons during two consecutive trains of APs (300 AP, $10 \mathrm{~Hz}$ ) in the presence of 2 or $4 \mathrm{mM}$ extracellular $\mathrm{Ca}^{2+}$.

containing Cav2.2, or in the intensity of Cav2.2 staining in presynaptic terminals (Supplementary Figures S2B,C). Nor did we find evidence for altered presynaptic localization and intensity of Cav2.1 (Supplementary Figures S2D-F or reduced density of presynaptic terminals (Supplementary Figure S2G) in DISC1 knockdown neurons. Thus, synaptic targeting and abundance of Cav2 channels does not seem to be regulated by DISC1.
We finally examined the effect of DISC1 on Cav2 currents by whole cell patch-clamp recordings. Co-transfection of recombinant DISC1 (Figure 6A) in HEK293T cells together with Cav2.2 and its auxiliary subunits (see "Materials and Methods" Section) resulted in a $27 \%$ increase in Cav2.2 peak current density (Figures 6B,C). Likewise, tail current density of Cav2.2 was substantially elevated in DISC1overexpressing cells (Figures 6D,E). Current density can be 

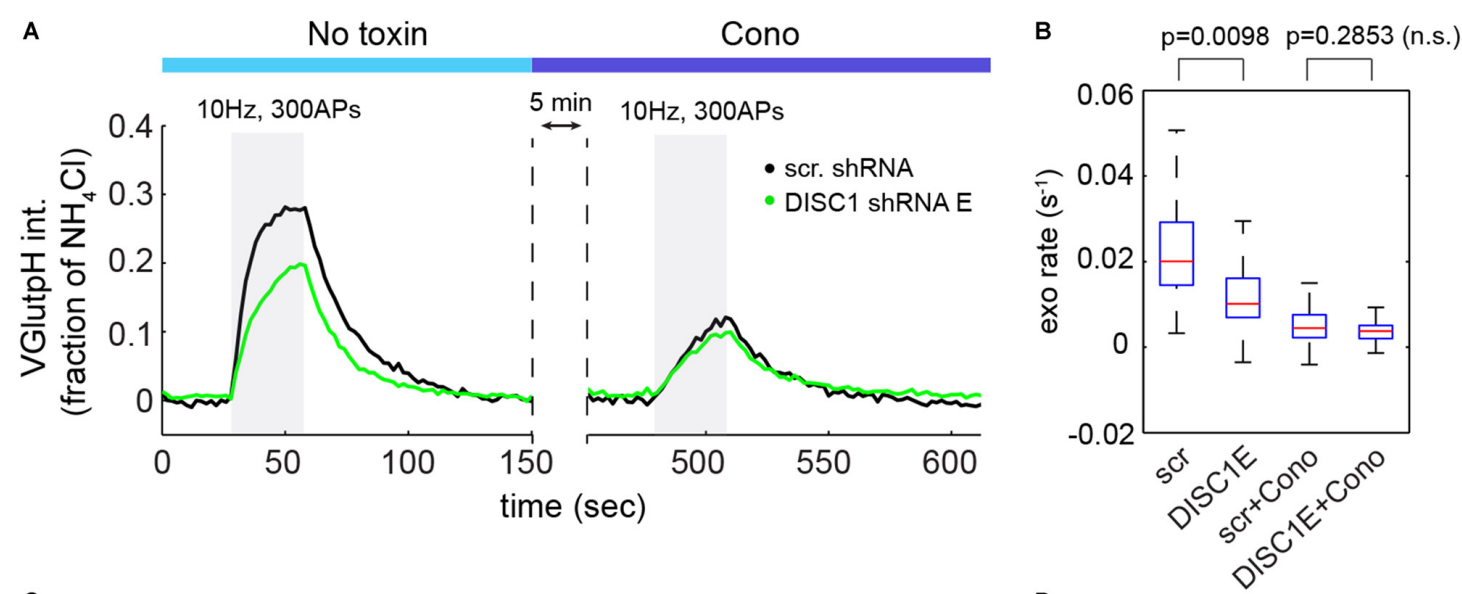

C

Aga

D
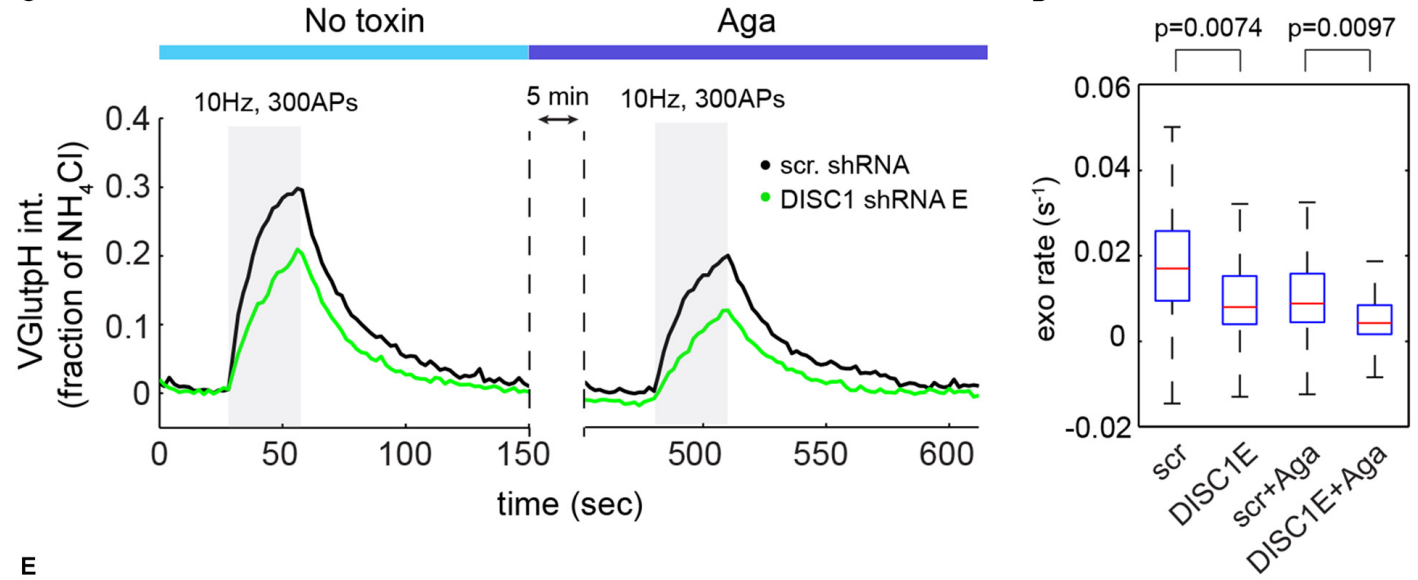

$\mathrm{E}$

\begin{tabular}{|c|c|c|}
\hline $\begin{array}{c}\text { \% inhibition of exo } \\
\text { rate by DISC1 KD }\end{array}$ & $\begin{array}{c}\text { before } \\
\text { toxin }\end{array}$ & $\begin{array}{c}\text { after } \\
\text { toxin }\end{array}$ \\
\hline Cono & $43.9 \%$ & $16.5 \%$ \\
\hline Aga & $43.4 \%$ & $49.5 \%$ \\
\hline
\end{tabular}

FIGURE 5 | DISC1 regulates Cav2.2-dependent SV exocytosis. (A) Average VGpH traces in scr (101 boutons, 6 fields, 2 exps) and DISC1-E (87 boutons, 5 fields, 2 exps) shRNA-expressing neurons during consecutive trains of APs (300 AP, $10 \mathrm{~Hz}$ ) in the absence or presence of the Cav2.2 blocker $\omega$-Conotoxin GVIA (125 nM). (B) Boxplot of SV exocytic rates before and after $\omega$-Conotoxin GVIA application. Exocytic rates were measured by linear fitting of the first six time points of the $\mathrm{vGpH}$ response. (C) Average $\mathrm{VGpH}$ traces in scr (1294 boutons, 9 fields, 3 exps) and DISC1-E (1282 boutons, 9 fields, 3 exps) shRNA-expressing neurons during consecutive trains of APs (300 AP, $10 \mathrm{~Hz}$ ) in the absence or presence of the Cav2.1 blocker $\omega$-Agatoxin TK (125 nM). (D) Boxplot of SV exocytic rates before and after $\omega$-Agatoxin TK application. (E) Table showing the percentage of inhibition of exocytosis rate by DISC1 knockdown before and after Cav2.2- or Cav2.1 blockade.

affected by a change in channel gating or by the number of channels at the cell surface. To test the first possibility, we examined the voltage-dependent properties of Cav2.2 current activation and saw no difference between control and DISC1-overexpressing cells (Figure 6F). This suggests that DISC1 promotes surface delivery and/or stabilize surface expression of Cav2.2. Recording of Cav2.1 currents revealed a similar, voltage-independent, potentiation effect of DISC1 (Figures 6G-K). Thus, DISC1 equally augments both Cav2.2 and Cav2.1 currents in this heterologous system, presumably by increasing surface expression of these $\mathrm{Ca}^{2+}$ channels. These results also imply the presence of an additional layer of regulation in hippocampal neurons that restricts the activity of DISC1 to Cav2.2-dependent SV exocytosis.

\section{DISCUSSION}

We used here two independent gene-targeting approaches together with large-scale imaging of presynaptic function to determine the impact of the mental disease gene DISC1 on the glutamate release machinery. Our results show that DISC1 accelerates SV exocytosis and thus enhances presynaptic performance. This boosting effect is mediated by N-type $\mathrm{Ca}^{2+}$ channels, establishing the first link between DISC1 and VGCC activity. 
A

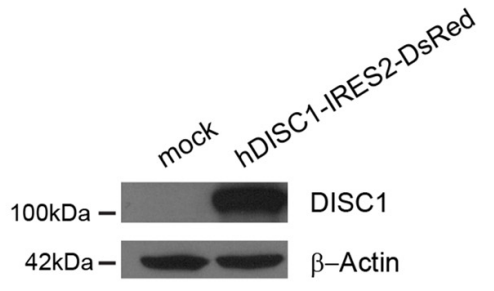

D

Cav2.2

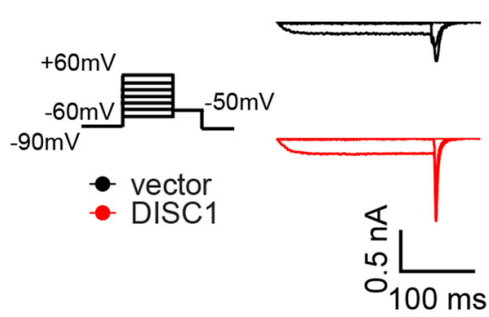

G

Cav2.1

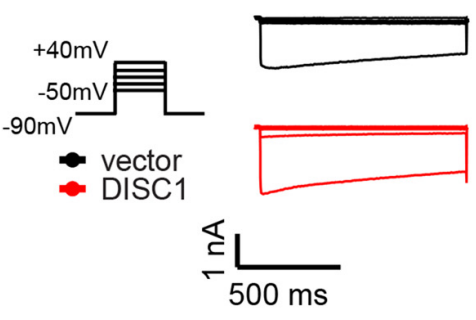

J

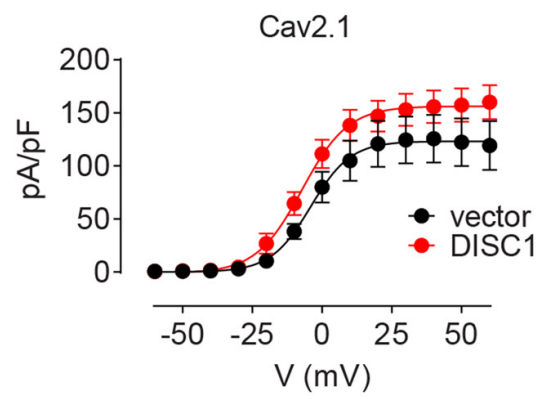

B

Cav2.2

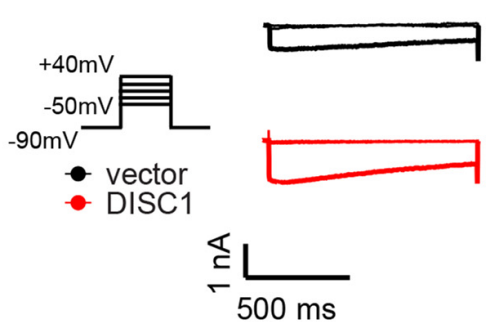

E

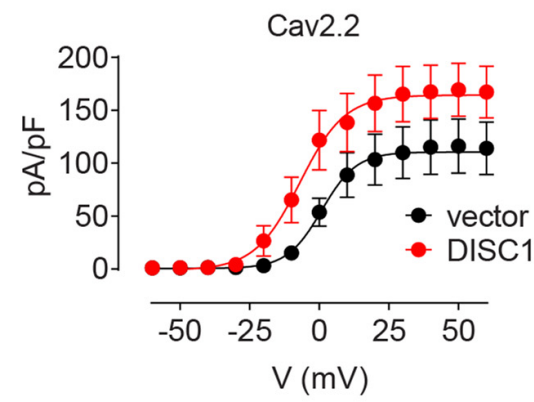

H

Cav2.1

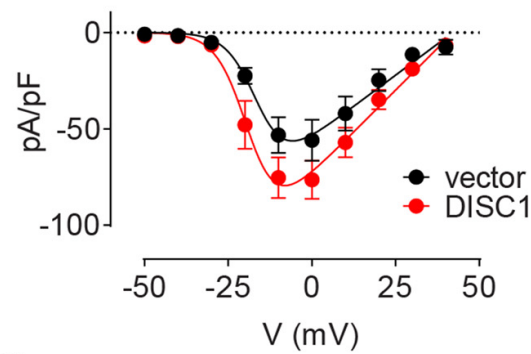

K

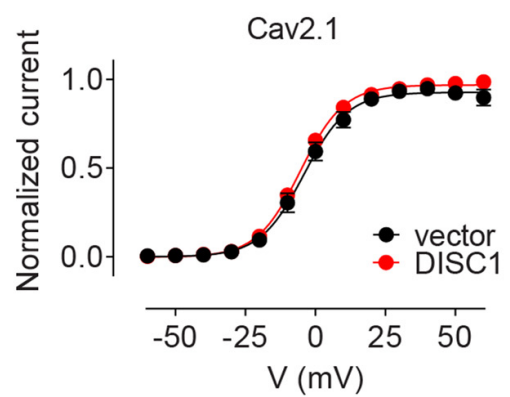

Cav2.2

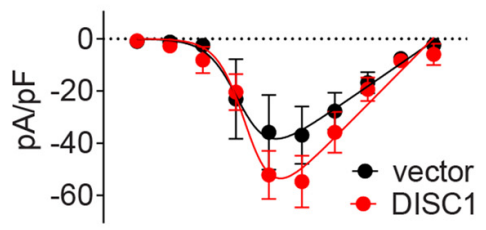

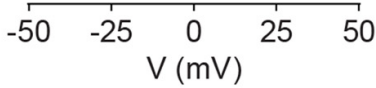

F

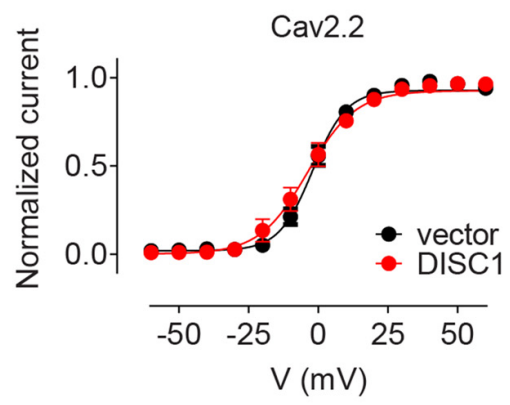

I

Cav2.1

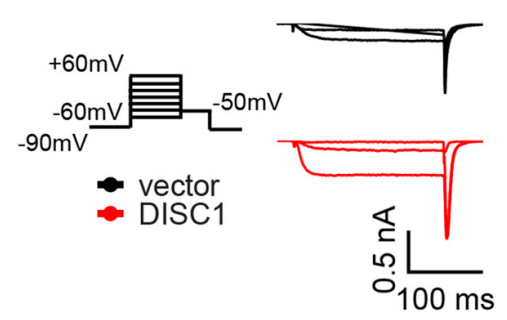

FIGURE 6 | DISC1 enhances Cav2.2 and Cav2.1 currents. (A) Western blot showing expression of ectopic (human) DISC1 in HEK293 cells. (B,C) Cav2.2 Current-voltage (I-V) curves for hDISC1-expressing and control cells. (B) Stimulation protocol and individual current responses shown at three different voltages $(-30,0$ and $30 \mathrm{mV}$ ) (C). Average I-V plots for hDISC1 (peak $=54.2 \pm 4.5$ pA/pF, 13 cells) and control (peak $=39.2 \pm 4.9$ pA/pF, 12 cells), $p=0.033$. (D-F) Cav2.2 activation curves in response to the tail protocol. (D) lllustration of the tail protocol and individual tail currents measured at $-50 \mathrm{mV}$ after three different voltage steps (-20, 0 and $40 \mathrm{mV}$ ). (E) Average current density based on tail currents for DISC1 (164.3 $\pm 7.3 \mathrm{pA} / \mathrm{pF}, 12$ cells) and control (110.5 $\pm 6.3 \mathrm{pA} / \mathrm{pF}, 12 \mathrm{cells}),{ }^{*} p<0.001$. (F) Normalized activation curve from tail currents showing no significant difference between DISC1 $\left(V_{50}:-6.98 \pm 3.43 \mathrm{mV}, 12 \mathrm{cells}\right)$ and control $\left(V_{50}: 0.86 \pm 3.62\right.$ $\mathrm{mV}, 12$ cells), $p=0.13$. (G,H) Cav2.1 Current-voltage (I-V) curves for hDISC1-expressing and control cells. (G) Stimulation protocol and individual current responses shown at three different voltages $(-30,0$ and $30 \mathrm{mV}$ ). (H) Average I-V plots for hDISC1 (peak $=79.9 \pm 8.3 \mathrm{pA} / \mathrm{pF}, 15$ cells) and control (peak $=56.3 \pm 7.4 \mathrm{pA} / \mathrm{pF}$, 13 cells), $p=0.046$. (I-K) Cav2.1 activation curves in response to the tail protocol. (I) Illustration of the tail protocol and individual tail currents measured at $-50 \mathrm{mV}$ after three different voltage steps $(-20,0$ and $40 \mathrm{mV}$ ). (J) Average current density based on tail currents for DISC1 (156.3 $\pm 5.7 \mathrm{pA} / \mathrm{pF}, 25 \mathrm{cells})$ and control $(123.2 \pm 7.4 \mathrm{pA} / \mathrm{pF}, 17 \mathrm{cells}),{ }^{*} p=0.003$. (K) Normalized activation curve from tail currents showing no significant difference between DISC1 ( $\mathrm{V}_{50}:-5.39 \pm 0.49$ $\mathrm{mV}, 24$ cells) and control ( $\mathrm{V}_{50}:-4.41 \pm 0.93 \mathrm{mV}, 17$ cells), $p=0.31$. 
DISC1 inactivation in hippocampal neurons results in decreased $\mathrm{Ca}^{2+}$ transients at hippocampal terminals in response to AP firing, consistent with a deficit in $\mathrm{Ca}^{2+}$ entry. Because these $\mathrm{Ca}^{2+}$ signals are shaped by both $\mathrm{Ca}^{2+}$ entry and clearance processes, we cannot exclude a role of DISC1 in regulating $\mathrm{Ca}^{2+}$ extrusion or buffering. A stimulatory effect of DISC1 on $\mathrm{Ca}^{2+}$ entry is further supported, however, by its positive impact on Cav2.2 and Cav2.1 currents, the two main VGCCs underlying the initiation of neurotransmission at hippocampal synapses. How DISC1 enhances Cav2 currents is unclear. The lack of an effect on gating suggests that DISC1 promotes surface expression of these $\mathrm{Ca}^{2+}$ channels, although we cannot exclude a role of DISC1 in regulating single-channel conductance or open probability. Our immunolocalization studies revealed no detectable impact of DISC1 on presynaptic abundance of Cav2 channels. This does not rule out, however, the possibility that DISC1 regulates the number of functional $\mathrm{Ca}^{2+}$ channels at the surface of the terminal.

Although overexpression of DISC1 enhances both Cav2.1 and Cav2.2 currents in a heterologous system, our findings point to a selective influence of DISC1 on Cav2.2-dependent SV exocytosis. How is this selectivity achieved? It is possible that both Cav2.1 and Cav2.2 compete for DISC1 regulation at presynaptic terminals. The dominant contribution of Cav2.2 to SV release probably results (at least in part) from increased levels of Cav2.2 (relative to Cav2.1) at hippocampal terminals (Ariel et al., 2013). Thus, preferential regulation of Cav2.2 by endogenous levels of DISC1 combined with greater abundance of Cav2.2 may explain why DISC1-dependent SV exocytosis appears to be exclusively mediated by Cav2.2. Specificity could also arise, however, from functions of DISC1 downstream of $\mathrm{Ca}^{2+}$ entry. For example, DISC1 could be involved in positioning Cav2.2 in close proximity to the SV release machinery at the active zone, a process that dictates speed and fidelity of neurotransmission. While the role of $\mathrm{Ca}^{2+}$ entry in SV exocytosis is undisputed, its impact on SV endocytosis remains somewhat controversial. Recent evidence suggests, however, that $\mathrm{Ca}^{2+}$ couples rates of SV exo- and endocytosis and optimizes endocytic rates during AP bursts (Armbruster et al., 2013). We observed reduced endocytic rates in both DISC1-silenced and DISC1 ${ }^{\Delta 2-3 / \Delta 2-3}$ neurons. Although this effect was small and did not reach statistical significance, our results suggest that the influence of DISC1 on Cav2.2 impacts both exo- and endocytosis of SVs at hippocampal terminals.

Our findings extend on two recent studies implicating DISC1 in presynaptic function. In glutamatergic neurons differentiated from human iPS cells and derived from members of a family with a frameshift mutation in DISC1, deficits in SV release were observed after $\mathrm{KCl}$-induced depolarization (Wen et al., 2014). In a separate report, RNAi knockdown of DISC1 in layer $2 / 3$ neocortical neurons increases pairedpulse facilitation and appears to reduce probability of glutamate release (Maher and Loturco, 2012). Collectively, these results clearly identify DISC1 as a positive modulator of glutamate release.
The efficacy of neurotransmitter release determines not only the strength of synaptic excitation, but also dictates various forms of short-term plasticity (Abbott and Regehr, 2004), suggesting broad functions of DISC1 in synaptic computation and neural circuit performance. Although we have not directly measured release probability $\left(\mathrm{P}_{\mathrm{r}}\right)$, the impact of DISC1 on $\mathrm{Ca}^{2+}$ entry and SV cycling suggests a positive influence on $\mathrm{P}_{\mathrm{r}}$ (see Maher and Loturco, 2012). Because synaptic facilitation and depression depend largely on the initial $\mathrm{P}_{\mathrm{r}}$ (high $\mathrm{P}_{\mathrm{r}}$ favors depression while low $\mathrm{P}_{\mathrm{r}}$ favors facilitation), our results predict altered shortterm plasticity in DISC1-deficient neural circuits. Of interest, abnormal short-term plasticity has been associated with deficits in moment-to-moment information processing and working memory, two hallmarks of schizophrenia (Crabtree and Gogos, 2014). Reduced efficacy in glutamate release may also be relevant for forms of long-term potentiation (LTP) that have clear presynaptic components, such as LTP at the perforant pathgranule cell synapse in the dentate gyrus (Errington et al., 1987). Notably, this form of LTP is impaired in DISC1 $1^{\Delta 2-3 / \Delta 2-3}$ mice-a stronger tetanic stimulus is required for the expression of LTP (Kuroda et al., 2011). Our findings suggest that this LTP deficit could be due to a failure of the presynaptic terminal to undergo activity-dependent increase in release probability.

Several genes encoding VGCC subunits, including CACNA1C, CACNB2 and CACNA1I have repeatedly been associated with schizophrenia and other psychiatric disorders (Ferreira et al., 2008; Cross-Disorder Group of the Psychiatric Genomics, 2013; Hamshere et al., 2013; Ripke et al., 2013; Schizophrenia Working Group of the Psychiatric Genomics, 2014). Although CACNA1A (Cav2.1) and CACNA1B (Cav2.2) are not typically associated with risk loci, RIM1 (also called RIMS1) - a presynaptic scaffold that regulates density of P/Q- and N-type $\mathrm{Ca}^{2+}$ channels and SV docking at the active zone (Han et al., 2011) - was recently identified as a candidate gene for schizophrenia in the largest GWAS study conducted to date (Schizophrenia Working Group of the Psychiatric Genomics, 2014). Together these findings point to neurotransmitter release as a central process targeted in schizophrenia.

In conclusion, our results shed light on a novel mechanism by which a major susceptibility gene for mental illness enhances the efficacy of glutamate release, and provide further support for a central role of glutamate neurotransmission in schizophrenia and other major mood disorders.

\section{AUTHOR CONTRIBUTIONS}

WT and JVT performed and analyzed all imaging and biochemical experiments. QL performed and analyzed whole-cell patch-clamp recordings. MB carried out the statistical analysis. KBL made the original observation implicating DISC1 in SV cycling. $\mathrm{KKu}$ and $\mathrm{KKa}$ made the DISC1 $\triangle 2-3$ mouse and the DISC1 C-terminus antibody. WT and MF wrote Matlab scripts. TWS and MF supervised the project. MF wrote the article. 


\section{ACKNOWLEDGMENTS}

We thank Bo Lu for critical reading of the manuscript. We are in debt to Durgadevi Alagappan and Tan Li-Ting for excellent technical assistance. This work was supported by a grant to MF from the Ministry of Education Academic Research Fund (MOE2013-T2-1-053).

\section{SUPPLEMENTARY MATERIAL}

The Supplementary Material for this article can be found online at: http://journal.frontiersin.org/article/10.3389/fnsyn. 2016.00015/abstract

FIGURE S1 | Variability of SV exocytic responses. Scatter plot showing SV exocytic rates in individual boutons across six (color-coded) independent preparations. Each preparation comprises three fields colored light to dark. These rates were measured in hippocampal neurons (DIV14-16) expressing scr shRNA and correspond to the data shown in Figure 2.

\section{REFERENCES}

Abbott, L. F., and Regehr, W. G. (2004). Synaptic computation. Nature 431, 796-803. doi: 10.1038/nature03010

Akerboom, J., Chen, T. W., Wardill, T. J., Tian, L., Marvin, J. S., Mutlu, S., et al. (2012). Optimization of a GCaMP calcium indicator for neural activity imaging. J. Neurosci. 32, 13819-13840. doi: 10.1523/jneurosci.260112.2012

Ariel, P., Hoppa, M. B., and Ryan, T. A. (2013). Intrinsic variability in Pv, RRP size, $\mathrm{Ca}^{2+}$ channel repertoire and presynaptic potentiation in individual synaptic boutons. Front. Synaptic Neurosci. 4:9. doi: 10.3389/fnsyn.2012. 00009

Ariel, P., and Ryan, T. A. (2010). Optical mapping of release properties in synapses. Front. Neural Circuits 4:18. doi: 10.3389/fncir.2010.00018

Armbruster, M., Messa, M., Ferguson, S. M., De Camilli, P., and Ryan, T. A. (2013). Dynamin phosphorylation controls optimization of endocytosis for brief action potential bursts. Elife 2:e00845. doi: 10.7554/elife. 00845

Burrone, J., Li, Z., and Murthy, V. N. (2006). Studying vesicle cycling in presynaptic terminals using the genetically encoded probe synaptopHluorin. Nat. Protoc. 1, 2970-2978. doi: 10.1038/nprot. 2006.449

Catterall, W. A., Leal, K., and Nanou, E. (2013). Calcium channels and shortterm synaptic plasticity. J. Biol. Chem. 288, 10742-10749. doi: 10.1074/jbc.r112. 411645

Chubb, J. E., Bradshaw, N. J., Soares, D. C., Porteous, D. J., and Millar, J. K. (2008). The DISC locus in psychiatric illness. Mol. Psychiatry 13, 36-64. doi: 10.1038/sj. mp.4002106

Clapcote, S. J., Lipina, T. V., Millar, J. K., Mackie, S., Christie, S., Ogawa, F., et al. (2007). Behavioral phenotypes of Discl missense mutations in mice. Neuron 54, 387-402. doi: 10.1016/j.neuron.2007.04.015

Crabtree, G. W., and Gogos, J. A. (2014). Synaptic plasticity, neural circuits and the emerging role of altered short-term information processing in schizophrenia. Front. Synaptic Neurosci. 6:28. doi: 10.3389/fnsyn.2014. 00028

Cross-Disorder Group of the Psychiatric Genomics, C. (2013). Identification of risk loci with shared effects on five major psychiatric disorders: a genome-wide analysis. Lancet 381, 1371-1379. doi: 10.1016/s0140-6736(12) 62129-1

Cross-Disorder Group of the Psychiatric Genomics, C., Lee, S. H., Ripke, S., Neale, B. M., Faraone, S. V., Purcell, S. M., et al. (2013). Genetic relationship between five psychiatric disorders estimated from genome-wide SNPs. Nat. Genet. 45, 984-994. doi: 10.1038/ng.2711
FIGURE S2 | Presynaptic localization and abundance of Cav2 channels are unaffected by DISC1 silencing. (A,D) Immunolocalization of endogenous Cav2.2 (A) or Cav2.1 (D) and bassoon in rat hippocampal neurons (DIV 14-15) expressing scr and DISC1-E shRNAs. Arrowheads indicate boutons positive for Cav2.2 and bassoon (A) or Cav2.1 and bassoon (D). Scale bar $10 \mu \mathrm{m}$. (B) Percentage of Cav2.2-positive boutons in scr (5 cells) and DISC1-E (5 cells) shRNA-expressing neurons. (C) Cav2.2 fluorescence intensity measured at individual presynaptic boutons (based on bassoon staining) in (B). (E) Percentage of Cav2.1-positive boutons in scr (8 cells) and DISC1-E (8 cells) shRNA-expressing neurons. (F) Cav2.1 fluorescence intensity measured at individual presynaptic boutons (based on bassoon staining) in (E). (G) Quantification of the density of presynaptic terminals (based on bassoon staining) in neurons expressing scr (24 cells), DISC1-E (21 cells) and -A (17 cells) shRNAs from four independent experiments.

TABLE T1 | Statistical analysis of synaptic responses. Column 1: datasets. Column 2: $p$ values derived from the field averaging approach, with each field weighted according to its number of boutons (see "Materials and Methods" Section). Column 3: $p$ values derived from the linear mixed model, where intra-field correlations and variations in neuron preparations are taken into account (see "Materials and Methods" Section).

Duan, X., Chang, J. H., Ge, S., Faulkner, R. L., Kim, J. Y., Kitabatake, Y., et al. (2007). Disrupted-In-Schizophrenia 1 regulates integration of newly generated neurons in the adult brain. Cell 130, 1146-1158. doi: 10.1016/j.cell.2007. 07.010

Ekelund, J., Hennah, W., Hiekkalinna, T., Parker, A., Meyer, J., Lonnqvist, J., et al. (2004). Replication of 1q42 linkage in Finnish schizophrenia pedigrees. Mol. Psychiatry 9, 1037-1041. doi: 10.1038/sj.mp.4001536

Ekelund, J., Hovatta, I., Parker, A., Paunio, T., Varilo, T., Martin, R., et al. (2001). Chromosome 1 loci in Finnish schizophrenia families. Hum. Mol. Genet. 10, 1611-1617. doi: 10.1093/hmg/10.15.1611

Errington, M. L., Lynch, M. A., and Bliss, T. V. (1987). Long-term potentiation in the dentate gyrus: induction and increased glutamate release are blocked by D(-)aminophosphonovalerate. Neuroscience 20, 279-284. doi: 10.1016/03064522(87)90019-4

Fernandez-Alfonso, T., and Ryan, T. A. (2004). The kinetics of synaptic vesicle pool depletion at CNS synaptic terminals. Neuron 41, 943-953. doi: 10 1016/s0896-6273(04)00113-8

Ferreira, M. A., O'donovan, M. C., Meng, Y. A., Jones, I. R., Ruderfer, D. M., Jones, L., et al. (2008). Collaborative genome-wide association analysis supports a role for ANK3 and CACNA1C in bipolar disorder. Nat. Genet. 40, 1056-1058. doi: $10.1038 / \mathrm{ng} .209$

Fromer, M., Pocklington, A. J., Kavanagh, D. H., Williams, H. J., Dwyer, S., Gormley, P., et al. (2014). De novo mutations in schizophrenia implicate synaptic networks. Nature 506, 179-184. doi: 10.1038/nature 12929

Galbraith, S., Daniel, J. A., and Vissel, B. (2010). A study of clustered data and approaches to its analysis. J. Neurosci. 30, 10601-10608. doi: 10.1523/jneurosci. 0362-10.2010

Garcia-Alvarez, G., Lu, B., Yap, K. A., Wong, L. C., Thevathasan, J. V., Lim, L., et al. (2015). STIM2 regulates PKA-dependent phosphorylation and trafficking of AMPARs. Mol. Biol. Cell 26, 1141-1159. doi: 10.1091/mbc.e1407-1222

Glessner, J. T., Reilly, M. P., Kim, C. E., Takahashi, N., Albano, A., Hou, C., et al. (2010). Strong synaptic transmission impact by copy number variations in schizophrenia. Proc. Natl. Acad. Sci. U S A 107, 10584-10589. doi: 10. 1073/pnas.1000274107

Hamshere, M. L., Walters, J. T., Smith, R., Richards, A. L., Green, E., Grozeva, D., et al. (2013). Genome-wide significant associations in schizophrenia to ITIH3/4, CACNA1C and SDCCAG8 and extensive replication of associations reported by the Schizophrenia PGC. Mol. Psychiatry 18, 708-712. doi: 10. 1038/mp.2012.67

Han, Y., Kaeser, P. S., Sudhof, T. C., and Schneggenburger, R. (2011). RIM determines $\mathrm{Ca}^{2+}$ channel density and vesicle docking at the 
presynaptic active zone. Neuron 69, 304-316. doi: 10.1016/j.neuron. 2010.12.014

Hikida, T., Jaaro-Peled, H., Seshadri, S., Oishi, K., Hookway, C., Kong, S., et al. (2007). Dominant-negative DISC1 transgenic mice display schizophreniaassociated phenotypes detected by measures translatable to humans. Proc. Natl. Acad. Sci. U S A 104, 14501-14506. doi: 10.1073/pnas.07047 74104

Howes, O., Mccutcheon, R., and Stone, J. (2015). Glutamate and dopamine in schizophrenia: an update for the 21st century. J. Psychopharmacol. 29, 97-115. doi: $10.1177 / 0269881114563634$

Huang, H., Tan, B. Z., Shen, Y., Tao, J., Jiang, F., Sung, Y. Y., et al. (2012). RNA editing of the IQ domain in $\mathrm{Ca}(\mathrm{v}) 1.3$ channels modulates their $\mathrm{Ca}^{2+}$ dependent inactivation. Neuron 73, 304-316. doi: 10.1016/j.neuron.2011. 11.022

Huettner, J. E., and Baughman, R. W. (1986). Primary culture of identified neurons from the visual cortex of postnatal rats. J. Neurosci. 6, 3044-3060.

International Schizophrenia, C., Purcell, S. M., Wray, N. R., Stone, J. L., Visscher, P. M., O'donovan, M. C., et al. (2009). Common polygenic variation contributes to risk of schizophrenia and bipolar disorder. Nature 460, 748-752. doi: 10. 1038/nature08185

Kaech, S., and Banker, G. (2006). Culturing hippocampal neurons. Nat. Protoc. 1, 2406-2415. doi: 10.1038/nprot.2006.356

Kamiya, A., Kubo, K., Tomoda, T., Takaki, M., Youn, R., Ozeki, Y., et al. (2005). A schizophrenia-associated mutation of DISC1 perturbs cerebral cortex development. Nat. Cell Biol. 7, 1167-1178. doi: 10.1038/ ncb1328

Kilpinen, H., Ylisaukko-Oja, T., Hennah, W., Palo, O. M., Varilo, T., Vanhala, R., et al. (2008). Association of DISC1 with autism and Asperger syndrome. Mol. Psychiatry 13, 187-196. doi: 10.1038/sj.mp.4002031

Kubo, K., Tomita, K., Uto, A., Kuroda, K., Seshadri, S., Cohen, J., et al. (2010). Migration defects by DISC1 knockdown in C57BL/6, 129X1/SvJ and ICR strains via in utero gene transfer and virus-mediated RNAi. Biochem. Biophys. Res. Commun. 400, 631-637. doi: 10.1016/j.bbrc.2010. 08.117

Kuroda, K., Yamada, S., Tanaka, M., Iizuka, M., Yano, H., Mori, D., et al. (2011). Behavioral alterations associated with targeted disruption of exons 2 and 3 of the Discl gene in the mouse. Hum. Mol. Genet. 20, 4666-4683. doi: 10. 1093/hmg/ddr400

Laird, N. M., and Ware, J. H. (1982). Random-effects models for longitudinal data. Biometrics 38, 963-974.

Lee, F. H., Fadel, M. P., Preston-Maher, K., Cordes, S. P., Clapcote, S. J., Price, D. J., et al. (2011). Discl point mutations in mice affect development of the cerebral cortex. J. Neurosci. 31, 3197-3206. doi: 10.1523/jneurosci.421910.2011

Li, H., Foss, S. M., Dobryy, Y. L., Park, C. K., Hires, S. A., Shaner, N. C., et al. (2011). Concurrent imaging of synaptic vesicle recycling and calcium dynamics. Front. Mol. Neurosci. 4:34. doi: 10.3389/fnmol.2011.00034

Maher, B. J., and Loturco, J. J. (2012). Disrupted-in-schizophrenia (DISC1) functions presynaptically at glutamatergic synapses. PLoS One 7:e34053. doi: 10.1371/journal.pone.0034053

Mao, Y., Ge, X., Frank, C. L., Madison, J. M., Koehler, A. N., Doud, M. K., et al. (2009). Disrupted in schizophrenia 1 regulates neuronal progenitor proliferation via modulation of GSK3beta/beta-catenin signaling. Cell 136, 1017-1031. doi: 10.1016/j.cell.2008.12.044

Miesenbock, G., De Angelis, D. A., and Rothman, J. E. (1998). Visualizing secretion and synaptic transmission with $\mathrm{pH}$-sensitive green fluorescent proteins. Nature 394, 192-195. doi: 10.1038/ 28190

Millar, J. K., Christie, S., Anderson, S., Lawson, D., Hsiao-Wei Loh, D., Devon, R. S., et al. (2001). Genomic structure and localisation within a linkage hotspot of Disrupted In Schizophrenia 1, a gene disrupted by a translocation segregating with schizophrenia. Mol. Psychiatry 6, 173-178. doi: 10.1038/sj.mp. 4000784

Millar, J. K., Pickard, B. S., Mackie, S., James, R., Christie, S., Buchanan, S. R., et al. (2005). DISC1 and PDE4B are interacting genetic factors in schizophrenia that regulate cAMP signaling. Science 310, 1187-1191. doi: 10.1126/science. 1112915
Millar, J. K., Wilson-Annan, J. C., Anderson, S., Christie, S., Taylor, M. S., Semple, C. A., et al. (2000). Disruption of two novel genes by a translocation co-segregating with schizophrenia. Hum. Mol. Genet. 9, 1415-1423. doi: 10. 1093/hmg/9.9.1415

Moskvina, V., Craddock, N., Holmans, P., Nikolov, I., Pahwa, J. S., Green, E., et al. (2009). Gene-wide analyses of genome-wide association data sets: evidence for multiple common risk alleles for schizophrenia and bipolar disorder and for overlap in genetic risk. Mol. Psychiatry 14, 252-260. doi: 10.1038/mp. 2008.133

Niwa, M., Kamiya, A., Murai, R., Kubo, K., Gruber, A. J., Tomita, K., et al. (2010). Knockdown of DISC1 by in utero gene transfer disturbs postnatal dopaminergic maturation in the frontal cortex and leads to adult behavioral deficits. Neuron 65, 480-489. doi: 10.1016/j.neuron.2010. 01.019

Pletnikov, M. V., Ayhan, Y., Nikolskaia, O., Xu, Y., Ovanesov, M. V., Huang, H., et al. (2008). Inducible expression of mutant human DISC1 in mice is associated with brain and behavioral abnormalities reminiscent of schizophrenia. Mol. Psychiatry 13, 173-186. doi: 10.1038/sj.mp.40 02079

Poon, V. Y., Goh, C., Voorhoeve, P. M., and Fivaz, M. (2014). High-content imaging of presynaptic assembly. Front. Cell Neurosci. 8:66. doi: 10.3389/fncel. 2014.00066

Ripke, S., O'dushlaine, C., Chambert, K., Moran, J. L., Kahler, A. K., Akterin, S., et al. (2013). Genome-wide association analysis identifies 13 new risk loci for schizophrenia. Nat. Genet. 45, 1150-1159. doi: 10.1038/ ng. 2742

Sachs, N. A., Sawa, A., Holmes, S. E., Ross, C. A., Delisi, L. E., and Margolis, R. L. (2005). A frameshift mutation in Disrupted in Schizophrenia 1 in an American family with schizophrenia and schizoaffective disorder. Mol. Psychiatry 10, 758-764. doi: 10.1038/sj.mp.4001667

Sankaranarayanan, S., De Angelis, D., Rothman, J. E., and Ryan, T. A. (2000). The use of pHluorins for optical measurements of presynaptic activity. Biophys. J. 79, 2199-2208. doi: 10.1016/s0006-3495(00) 76468-x

Schizophrenia Working Group of the Psychiatric Genomics, C. (2014). Biological insights from 108 schizophrenia-associated genetic loci. Nature 511, 421-427. doi: 10.1038/nature 13595

Shen, S., Lang, B., Nakamoto, C., Zhang, F., Pu, J., Kuan, S. L., et al. (2008). Schizophrenia-related neural and behavioral phenotypes in transgenic mice expressing truncated Disc1. J. Neurosci. 28, 10893-10904. doi: 10. 1523/jneurosci.3299-08.2008

Shinoda, T., Taya, S., Tsuboi, D., Hikita, T., Matsuzawa, R., Kuroda, S., et al. (2007). DISC1 regulates neurotrophin-induced axon elongation via interaction with Grb2. J. Neurosci. 27, 4-14. doi: 10.1523/jneurosci.382506.2007

Singh, K. K., Ge, X., Mao, Y., Drane, L., Meletis, K., Samuels, B. A., et al. (2010). Dixdc1 is a critical regulator of DISC1 and embryonic cortical development. Neuron 67, 33-48. doi: 10.1016/j.neuron.2010. 06.002

St Clair, D., Blackwood, D., Muir, W., Carothers, A., Walker, M., Spowart, G., et al. (1990). Association within a family of a balanced autosomal translocation with major mental illness. Lancet 336, 13-16. doi: 10.1016/0140-6736(90) 91520-k

Steinecke, A., Gampe, C., Valkova, C., Kaether, C., and Bolz, J. (2012). Disrupted-in-Schizophrenia 1 (DISC1) is necessary for the correct migration of cortical interneurons. J. Neurosci. 32, 738-745. doi: 10.1523/jneurosci.503611.2012

Thevathasan, J. V., Tan, E., Zheng, H., Lin, Y. C., Li, Y., Inoue, T., et al. (2013). The small GTPase HRas shapes local PI3K signals through positive feedback and regulates persistent membrane extension in migrating fibroblasts. Mol. Biol. Cell 24, 2228-2237. doi: 10.1091/mbc.E12-12-0905

Tian, L., Hires, S. A., Mao, T., Huber, D., Chiappe, M. E., Chalasani, S. H., et al. (2009). Imaging neural activity in worms, flies and mice with improved GCaMP calcium indicators. Nat. Methods 6, 875-881. doi: 10.1038/ nmeth.1398

Tiscornia, G., Singer, O., and Verma, I. M. (2006). Production and purification of lentiviral vectors. Nat. Protoc. 1, 241-245. doi: 10.1038/nprot. 2006.37 
Tsuboi, D., Kuroda, K., Tanaka, M., Namba, T., Iizuka, Y., Taya, S., et al. (2015). Disrupted-in-schizophrenia 1 regulates transport of ITPR1 mRNA for synaptic plasticity. Nat. Neurosci. 18, 698-707. doi: 10.1038/ nn.3984

Voglmaier, S. M., Kam, K., Yang, H., Fortin, D. L., Hua, Z., Nicoll, R. A., et al. (2006). Distinct endocytic pathways control the rate and extent of synaptic vesicle protein recycling. Neuron 51, 71-84. doi: 10.1016/j.neuron.2006. 05.027

Wen, Z., Nguyen, H. N., Guo, Z., Lalli, M. A., Wang, X., Su, Y., et al. (2014). Synaptic dysregulation in a human iPS cell model of mental disorders. Nature 515, 414-418. doi: 10.1038/nature13716
Conflict of Interest Statement: The authors declare that the research was conducted in the absence of any commercial or financial relationships that could be construed as a potential conflict of interest.

Copyright (c) 2016 Tang, Thevathasan, Lin, Lim, Kuroda, Kaibuchi, Bilger, Soong and Fivaz. This is an open-access article distributed under the terms of the Creative Commons Attribution License (CC BY). The use, distribution and reproduction in other forums is permitted, provided the original author(s) or licensor are credited and that the original publication in this journal is cited, in accordance with accepted academic practice. No use, distribution or reproduction is permitted which does not comply with these terms. 